\title{
On the Flexibility of Electricity Consumers: Introducing Notice Time
}

\author{
Lars Herre \\ School of Electrical Engineering \\ KTH Royal Institute of Technology \\ Stockholm, Sweden \\ Email: lars.herre@ee.kth.se
}

\author{
Lennart Söder \\ School of Electrical Engineering \\ KTH Royal Institute of Technology \\ Stockholm, Sweden \\ Email: lennart.soder@ee.kth.se
}

\begin{abstract}
Power systems with a large share of inherently intermittent energy sources require new approaches to system operation. Demand side management is seen as one potential resource for maintaining power balance in a future energy system with large amounts of volatile renewable energy generation. Existing demand response programs require flexibility in a set time frame from the electricity consumers in reaction to economic incentives. For a more detailed qualitative analysis of consumer flexibility, not only price but also notice time are imperative parameters. Former has been studied in numerous references whereas the impact of the latter has not yet been examined in depth. This paper presents a review of existing demand response programs and reflects on consumers' flexibility with respect to notice time. While some schemes include fixed notification schedules, others allow for variable notice time, or even continuous updating. In this paper, the consequences of notice time and update intervals on flexible electricity consumers are studied in Demand Bidding model with continuous forecast updates.
\end{abstract}

Index Terms-smart grids, power system simulation, power generation planning, power system economics, wind energy integration.

\section{INTRODUCTION}

Limited energy resources in the form of fossil fuels at a time when a paradigm shift in energy policy is occurring will characterise this century by intensified competition of renewable energy sources. Renewable energy from wind power has both a competitive and comparative advantage [1] and is seen as one of the most promising and mature large scale renewable alternatives in a future energy system. There are, however, also challenges related to the exploitation of wind energy. Due to the intermittent nature of wind power the power output is inherently volatile. Additionally, the limited predictability of wind speeds results in uncertain wind power forecasts. In a power system however, the balance of production and consumption needs to be balanced at all times. Any deviation may potentially result in a blackout, which is associated to high costs for society.

Several options exist to ensure the power balance in the long and short term. In a future smart grid, flexible electricity consumers are seen as one option of maintaining the power system in balance. The European Wind Energy Association concludes in [1] that for improved wind power integration "[...] distribution grids must be more actively managed". Demand
Response (DR) covers several aspects that are today provided by generation units or storage. Flexible consumers may replace or add on to these features in the future and add further possibilities to the scope of system services.

In [2] the potential of DR in Germany was addressed and its value for wind integration was estimated. In [3] the potential of different DR tariffs for wind integration were analysed. The National Renewable Energy laboratory addressed the utilization of DR for wind integration in [4]. The impacts of wind energy and DR on the day-ahead market were studied in [5]. In [6] DR was found to have highest impact during peak load hours with low wind power. From the converge analysis of wind power and DR in [7], it was figured that load aggregators must be able to bid foreseen ability to provide ancillary services into the day ahead and intraday markets. Demand shifting and peak clipping for wind integration were studied in [8]. [9] proposed a two-part compound bidding mode for wind power integration. A coupon-based DR model considering uncertain wind power forecasts was presented in[10] and both deficit-limiting scheduling and predicting based DR for wind integration were examined in [11]. The integration of variable wind energy using Demand Response including a utility function has been adressed in [12].

However, the above studies are based on Demand Response programs and models which are bound to a fixed time interval to notify consumers. None of these studies have studied the impact of different notice times of flexible consumers.

For enhanced integration of volatile renewable energy sources such as wind power, the notice time is an imperative parameter, since forecasts tend to become better as the target hour is approached. An exemplary illustration of the wind power forecast root mean square error over the forecast horizon in German control areas is shown in Fig. 1. The second graph of Fig. 1 illustrates the development of the probability distribution function of forecast errors corresponding to the forecast horizon. For simplicity, a normal distribution with a mean of 0 is illustrated.

The framework to analyse the influencing factors of flexible consumers is strongly bound to the electricity market set-up; the type of DR program [14], market price formulation and success indicators. It is logical that the flexibility of electricity consumers decreases as the notice time $\left(t_{n}\right)$ becomes shorter 

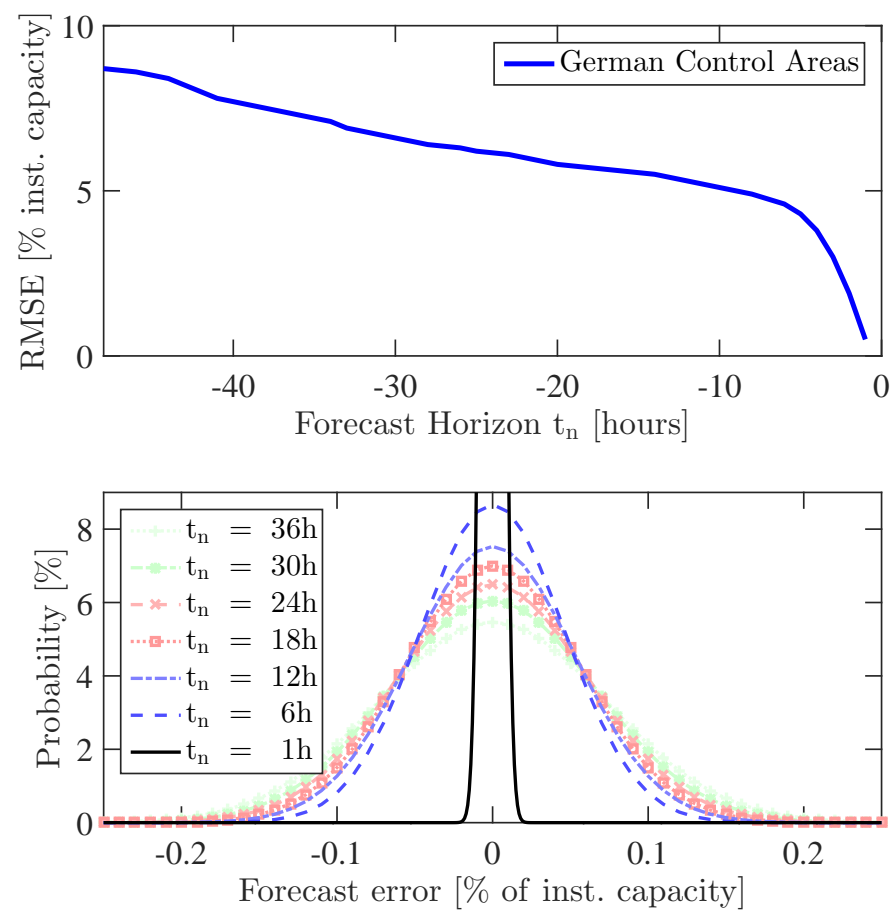

Fig. 1. Wind power forecast root mean square error (RMSE) and probability density function for selected forecast horizons (data from [13])

in most cases. On the other hand, forecasts of wind power production become more accurate the closer they approach the target hour. The question is thus, which consequences notice time and update intervals for improved forecasts have on the consumer participation. In order to formulate and solve this problem, a suitable model of DR is imperative. Some DR programs inherently include a fixed notification schedule, while others allow for a study of the effects of notifying and updating consumers.

Section II gives a review on existing DR schemes and judges their suitability for a notice time dependent model. In Section III an economic dispatch model for a subcategory of Demand Bidding (DB) is proposed that allows for the analysis of notice time using update intervals. A case study is presented in section IV. In section $\mathrm{V}$ the results of the case study are discussed and section VI gives a conclusion of this paper.

\section{Classification of Demand Response Programs}

The U.S. Department of Energy defines demand response as changes in electric usage by end-use customers from their normal consumption patterns in response to changes in the price of electricity over time, or to programs designed to induce lower electricity use at times of high wholesale market prices or when system reliability is jeopardized [15].

In the rapidly growing diversity of demand response programs, [14] proposed a classification of DR and summarized existing programs. This section gives a brief overview of Demand Response programs and possible classifications and definitions.
Three basic types of proposed classifications can be identified; market, dispatchability and time oriented classifications. With respect to this study, the most purposeful way of classifying DR programs is to cluster them by the time when the information for providing a certain service is exchanged. Fig. 2 shows the time frame of existing DR programs as classified in [15].

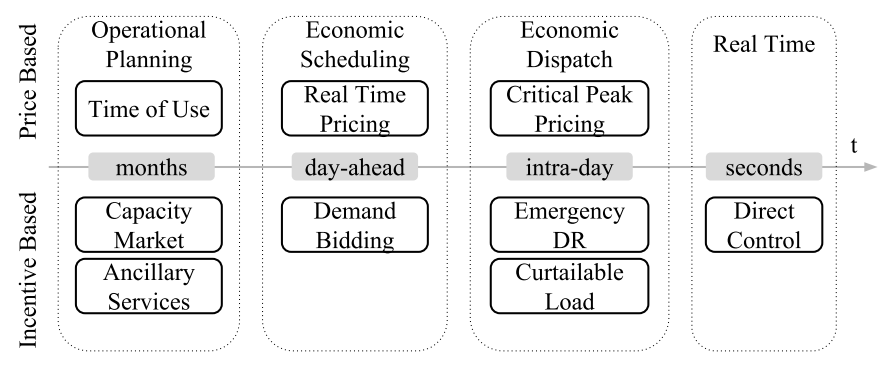

Fig. 2. Classification of DR by time

It is obvious that for the system operator, a very quick decision before the target hour, such as Direct Control, is optimal, since the most accurate wind power forecast is available only then. From the consumer point of view however, a reliable predetermined tariff is the easiest option. Such tariffs occur in Time of Use, Capacity Market, and Ancillary Service programs.

\section{Modeling Notice Time}

It can be concluded from Fig. 2, that only Demand Bidding (DB) and Real Time Pricing (RTP) allow for flexible economic scheduling starting on the day-ahead (DA). In order to examine consumer flexibility with respect to notice time, a continuously updated Demand Bidding (CU-DB) approach was developed in this study. The conceptual framework is borrowed from the Spanish intraday market, where new bids and offers are traded in update intervals $T_{u}$ of 6 hours. Every trading period is modeled to occur at a new market place, i.e. energy is traded anew independent of the previous contracts. The fact that most customers do not have access to the market due to a minimum bid size is taken care of by an aggregator. Furthermore, perfect competition and information are assumed.

\section{A. CU-DB Program}

The proposed model is illustrated in Fig. 3, starting at 12:00 DA for target hour 24 on the consecutive day. Every 6 hours, an updated wind power forecast $W_{f u}$ results in trading of additional energy at a new market place and rescheduling of flexible generation $\left(G_{u}\right)$ and consumption $\left(D_{f u}\right)$ units: The slope of the consumer's utility curve is assumed to increase by a factor $k_{u}$ as notice time decreases. The market equilibrium after the update is found at the intersection of the updated utility curves at price level $\lambda_{u}$. Directly before the target hour, a last market place determines the final equilibrium for the target hour, throughout which wind power is assumed constant. Upon a forecast update, energy is traded anew on the next 


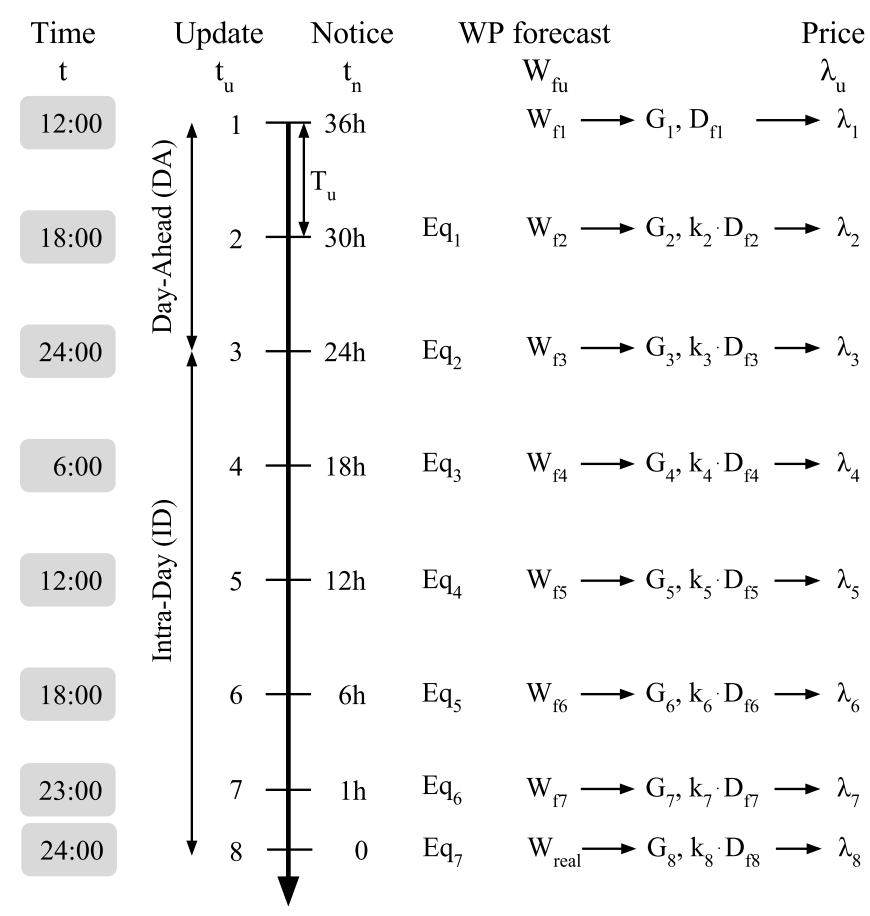

Fig. 3. Continuously Updated Demand Bidding (CUDB) model

market place, with the demand bid curve starting from the previous equilibrium.

At the $1^{\text {st }}$ update time $t_{u}=1$, the notice time $t_{n_{1}}$ is 36 hours with respect to target hour 24 . The forecasted price $\lambda_{1}$ is determined at 12:00 DA, based on the initial wind power forecast $W_{f 1}$, the cost of required conventional generation $G_{1}$ and initial flexible demand bidding curve $D_{f 1}$. The equilibria of three exemplary consecutive notice times are illustrated in Figs. 4 and 5.

Upon forecast update, the updated bid curves are merged at a new market place starting from the equilibrium of the previous update. Any additional generation and consumptio is traded independent of the previous market place. In th: way, both producers and consumers contribute to settle an foreseeable imbalance at an early notice time.

\section{B. Change of Wind Power Forecasts}

When approaching the target hour, wind power forecasi improve in accuracy. At the $2^{\text {nd }}$ notice time, at e.g. 18:0 $\mathrm{DA}$, the new wind power forecast $W_{f 2}$ is used to determin the price for additional flexible power generation $G_{2}$. Flexibl consumers can be seen as generation units with negativ production that are competing with conventional generators.

1) Decreasing Wind Power Forecasts: An illustration c a decreasing wind power forecast over three update steps is depicted in Fig. 4. In case of a lower wind power forecast, the flexible generation units may increase their scheduled production by $\Delta G_{u p}$. This could however also be provided by down-regulating consumers, that decrease their consumption by selling previously acquired energy $\Delta D_{\text {down }}$. The equilibria $E q_{u}$ at update $u$ are indicated in the inset.

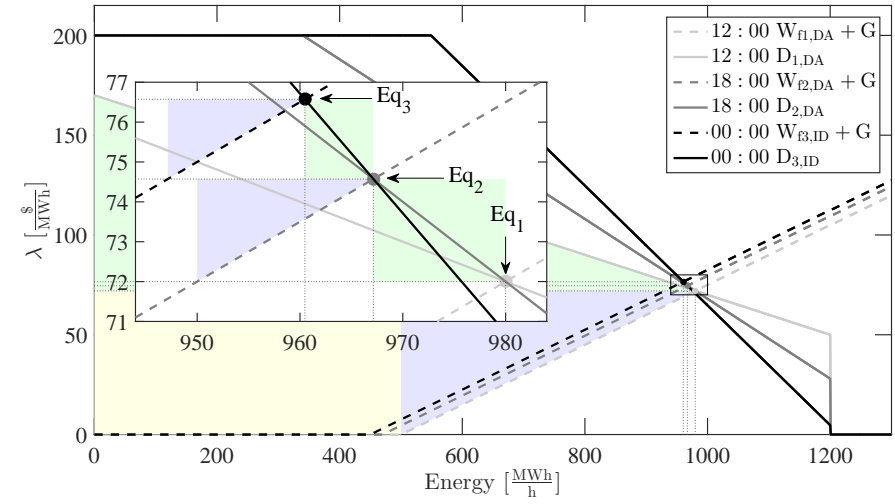

Fig. 4. $\Delta G_{u p}$ and $\Delta D_{\text {down }}$ upon updated forecast in CU-DB

The surplus of wind power producers (yellow), flexible generation (blue) and demand (green) at the $1^{\text {st }}$ notice time is shown. Further, the additional surplus $S$ of flexible generation (blue) and demand (green) at the respective update interval is illustrated in the inset of Fig. 4 and can be calculated by

$$
\begin{gathered}
S_{G, u p, j}=\frac{1}{2} \cdot \Delta G_{u p, i j} \cdot \Delta \lambda_{i j} \\
S_{D, \text { down }, j}=\Delta D_{\text {down }, i j} \cdot \Delta \lambda_{i j},
\end{gathered}
$$

where $j$ is the current and $i$ is the previous notice time

2) Increasing Wind Power Forecasts: An example of an increasing wind power forecast over three update steps is illustrated in Fig. 5. In case of a higher wind power forecast, the flexible generation units may produce less, i.e purchase previously sold energy $\Delta G_{\text {down }}$ for a lower price. This could however also be provided by up-regulating consumers, that increase their consumption $\Delta D_{\text {up }}$. For illustration purposes the initial slope $b_{D_{1}}=-0.1 \frac{\$}{M W h}$ is multiplied by a factor $k_{u}=t_{u}$ at each update time $t_{u}$ which decreases the flexibility of demand significantly. A summary of elasticity studies is found in e.g. [16].

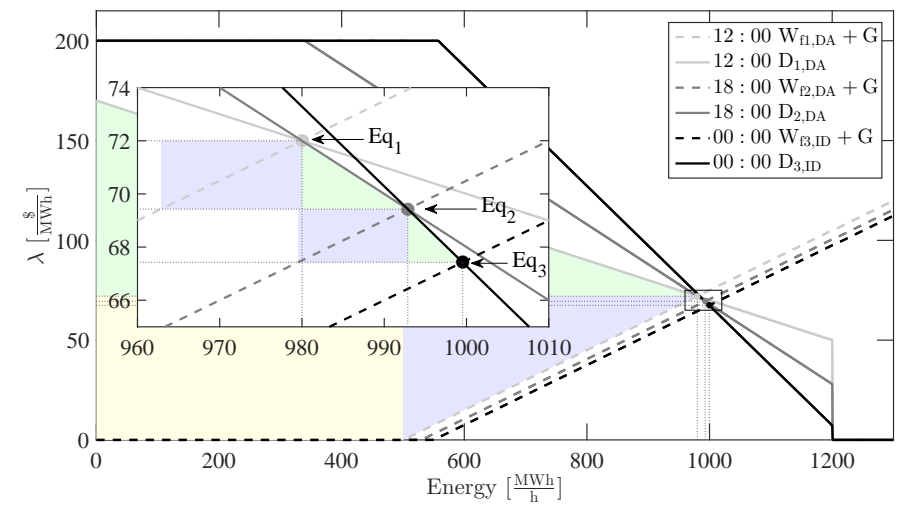

Fig. 5. $\Delta G_{\text {down }}$ and $\Delta D_{\text {up }}$ upon updated forecast in CU-DB

The additional surplus of flexible generation (blue) and demand (green) in the respective update interval is illustrated in Fig. 5 and can be calculated by

$$
S_{G, \text { down }, j}=\Delta G_{\text {down }, i j} \cdot \Delta \lambda_{i j}
$$




$$
S_{D, u p, j}=\frac{1}{2} \cdot \Delta D_{u p, i j} \cdot \Delta \lambda_{i j}
$$

\section{Cost Functions}

The flexible generation $G$ is modeled by a linear cost function as shown in Figs. 5 and 4 and flexible demand $D_{f}$ is modeled by a linear cost function with limits on maximal price and consumption. In addition, the elasticity of flexible consumers decreases with decreasing notice time, i.e. the slope of the utility function becomes steeper at every update interval. The demand is assumed to be deterministic, i.e. the actual load at the target hour is exactly the sum of purchased energy during the previous notice times. Due to the continuous trading in several updates and deterministic consumers, any imbalance is assumed to be settled in forward markets in this CU-DB scheme.

The overall surplus of flexible demand and generation can then be obtained by summing the surplus obtained in the markets of all update times:

$$
\begin{aligned}
& S_{G, \text { total }}=\sum_{u} S_{G, u} \\
& S_{D, \text { total }}=\sum_{u} S_{D, u} \\
& \text { IV. CASE STUDY }
\end{aligned}
$$

In this section, a selection of six particular scenarios is analysed. Table I summarizes the development of wind power forecasts from 36 hours ahead until the actual target hour 24. The first scenarios S1 and S2 describe a constant maximal over

TABLE I

SCENARIOS OF WIND POWER FORECAST DEVELOPMENT [ $\left.\frac{\mathrm{MWh}}{\mathrm{h}}\right]$

\begin{tabular}{l|rrrrrr}
\hline Scenario & S1 & S2 & S3 & S4 & S5 & S6 \\
\hline 12:00 DA & 537 & 463 & 537 & 463 & 500 & 500 \\
18:00 DA & 534 & 466 & 466 & 534 & 534 & 466 \\
24:00 DA & 531 & 469 & 531 & 469 & 531 & 469 \\
06:00 ID & 529 & 471 & 471 & 529 & 529 & 471 \\
12:00 ID & 527 & 473 & 527 & 473 & 527 & 473 \\
18:00 ID & 523 & 477 & 477 & 523 & 523 & 477 \\
23:00 ID & 502 & 498 & 502 & 498 & 502 & 498 \\
24:00 ID & 500 & 500 & 500 & 500 & 500 & 500 \\
\hline
\end{tabular}

and under estimate respectively. S3 and S4 describe scenarios where over and under estimate are constantly changing. S5 and S6 are similar to the first two scenarios, with the exception that the initial wind power forecast proves to be the the real production. In the reference scenario all updates forecast the real wind power. All above values of wind power forecasts are within a confidence range depicted in Fig. 6 and according to the error in Fig. 1.

\section{A. Results}

The wind power forecast $\left(W_{f}\right)$, the resulting equilibrium generation $\left(G_{f}\right)$, demand $\left(D_{f}\right)$ and price for the above scenarios are summarized in Fig. 7. It can be seen that prices are affected by both quantity of wind power deviation and notice

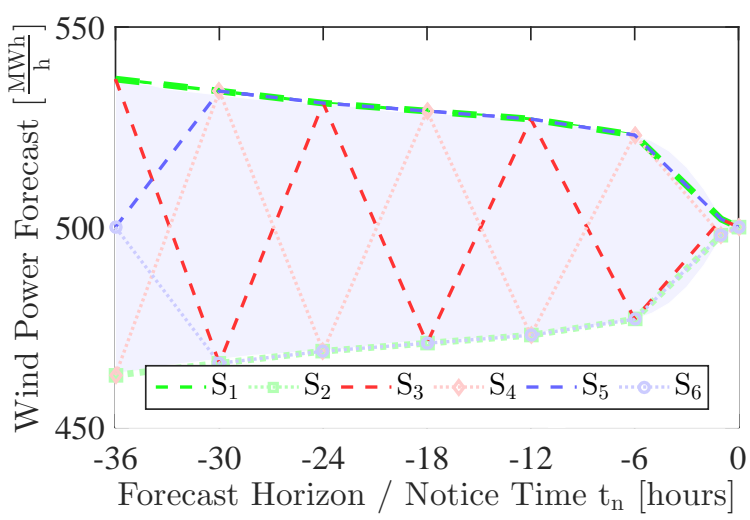

Fig. 6. Range of wind power forecast uncertainty in Scenarios S1 to S6

time. In scenarios S3 and S4 with highly fluctuating forecasts, the prices vary with larger magnitude during the first notice times, when the quantity of forecast deviations is large. A notable slope at the $7^{\text {th }}$ update (at notice time $t_{n}=1 \mathrm{~h}$ ) can be identified throughout all six scenarios. The reason is that the $1 \mathrm{~h}$ forecast is significantly better than the $6 \mathrm{~h}$ forecast, as was shown in Fig. 1. The price deviation in the last update that characterizes the real wind power production has less impact on the price level compared the the $7^{\text {th }}$ update.

\section{DISCUSSION}

This CU-DB model enables customers to be updated with a defined time period (e.g. $6 \mathrm{~h}$ as in Spain) on the current forecast of wind power production. Flexible consumers can participate in competition with flexible generation units in several forward markets.

This opens the opportunity for both bidding parties to increase their utility in the consecutive market places. However, power producers exploiting market power may strategically bid with a lowered wind power forecast in the first market, thus increasing their total surplus.

Flexible consumers are modelled with a linear bid curve whose slope increases the shorter the notice time. Through such model, the risk of the deterministic consumers is minimized, since they would only change their consumption if their overall cost can be minimized, but otherwise would stick to the initial consumption schedule. If consumers were not deterministic, an additional stochastic component would be introduced.

\section{CONCLUSION}

In this paper, the consequences of notice time and update intervals on flexible electricity consumers are studied. The review of Demand Response programs concluded that variations of Real Time Pricing (RTP) and Demand Bidding (DB) qualify for an analysis of notice time. A continuously updated Demand Bidding (CU-DB) model with consecutive wind power forecast updates was developed. The model was applied to study quantitatively the impact of notice time and uncertain wind power forecasts on flexible consumers' participation. In our 

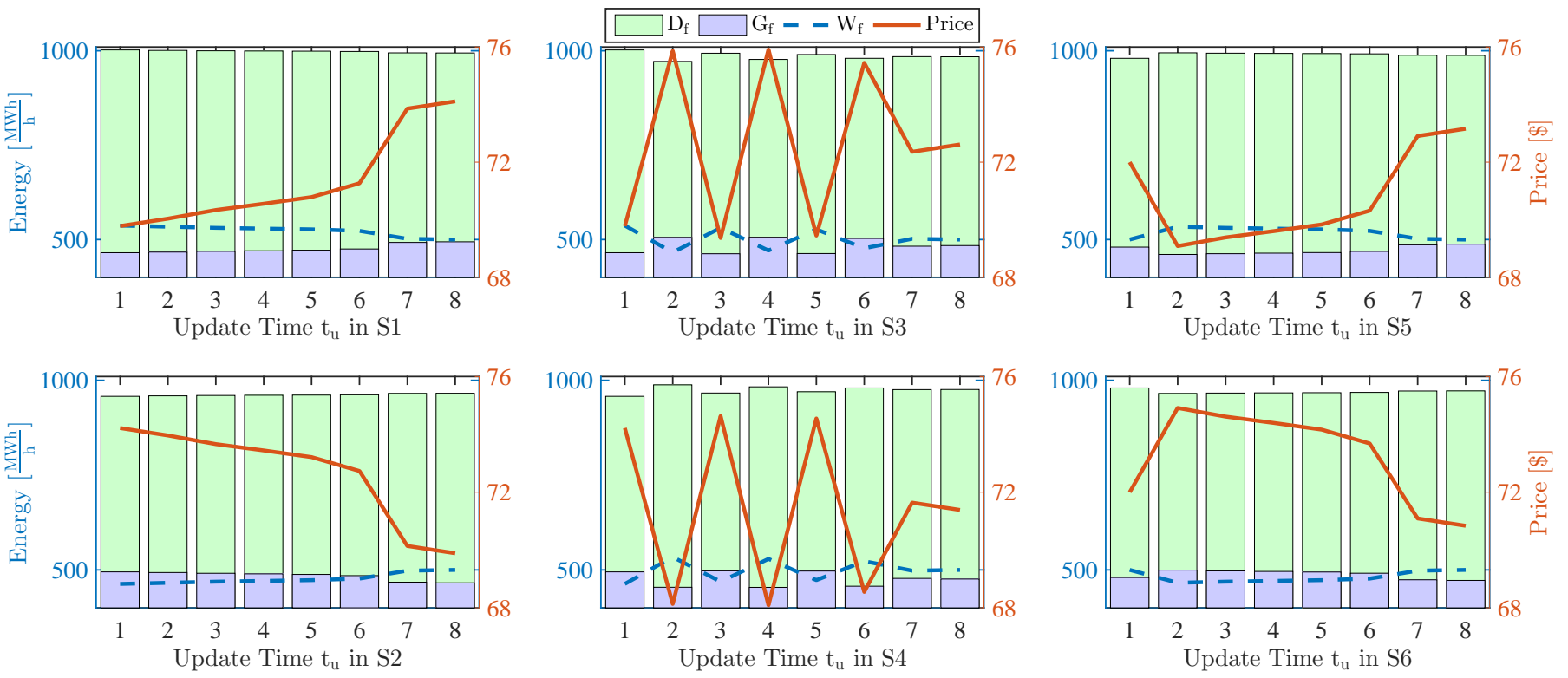

Fig. 7. Simulation results of the six scenarios for 8 consecutive notice times

CU-DB model, both forecast error magnitude and notice time affect the market equilibrium.

On the day-ahead (i.e. with long notice time), wind power forecasts show stochastically higher forecast deviations, while energy demand is more flexible in the scheduled consumption. In the intra-day frame (i.e with short notice time), the wind power forecasts tend to display smaller deviations, whereas energy demand is less flexible to reschedule consumption. In this study, we have shown how to analyse these two imperative parameters in a quantitative way.

A selection of six scenarios representing extreme wind power forecast deviation developments were studied and the results with respect to equilibrium demand and price were presented. It was found that an update with a short notice time can be beneficial to settle the deviation from earlier forecasts. This is due to the increasing certainty as forecasts approach the target hour.

\section{REFERENCES}

[1] E. W. E. A. (EWEA), "Large Scale Integration of Wind Energy in the European Power Supply: Analysis Issues and Recommendations," European Wind Energy Association (EWEA), Tech. Rep., 2005.

[2] M. Klobasa, "Analysis of demand response and wind integration in Germany's electricity market," IET Renewable Power Generation, vol. 4, no. 1, p. 55, 2010.

[3] P. Finn, C. Fitzpatrick, and M. Leahy, "Increased Penetration of Wind Generated Electricity using Real time Pricing \& Demand Side Management," IEEE International Symposium on Sustainable Systems and Technology, pp. 1-6, 2009.

[4] M. Milligan and B. Kirby, "Utilizing Load Response for Wind and Solar Integration and Power System Reliability," in The Windpower 2010 Conference \& Exhibition, no. July, 2010, pp. 1-21. [Online]. Available: http://www.nrel.gov/docs/fy10osti/48247.pdf

[5] D. Steen, P. Balram, L. A. Tuan, L. Reichenberg, and L. B. Tjernberg, "Impact Assessment of Wind Power and Demand Side Management on Day-Ahead Market Prices," in Innovative Smart Grid Technologies Conference Europe (ISGT-Europe), Istanbul, 2014, pp. 1-6.
[6] P. S. Moura and A. T. de Almeida, "The role of demandside management in the grid integration of wind power," Applied Energy, vol. 87, no. 8, pp. 2581-2588, 2010. [Online]. Available: http://linkinghub.elsevier.com/retrieve/pii/S0306261910000838

[7] S. Parkinson, D. Wang, and N. Djilali, "Toward low carbon energy systems: The convergence of wind power, demand response, and the electricity grid," IEEE PES Innovative Smart Grid Technologies, no. October, pp. 1-8, 2012. [Online]. Available: http://ieeexplore.iee.org/lpdocs/epic03/wrapper.htm?arnumber=6303401

[8] K. Dietrich, J. M. Latorre, A. Member, L. Olmos, and A. Ramos, "Demand Response in an Isolated System With High Wind Integration," IEEE Transactions on Power Systems, vol. 27, no. 1, pp. 20-29, 2012.

[9] Y. Xiao, X. Wang, C. Dang, C. Du, S. Member, and a. P. Consumers, "Two-part Compound Bidding Mode of Wind Power Considering Demand-side Interaction," in International Conference on Power System Technology (POWERCON), no. Powercon, 2014, pp. 20-22.

[10] X. Fang, Q. Hu, F. Li, B. Wang, and Y. Li, "Coupon-Based Demand Response Considering Wind Power Uncertainty: A Strategic Bidding Model for Load Serving Entities," IEEE Transactions on Power Systems, pp. 1-13, 2015.

[11] N. Çiçek and H. Deliç, "Demand Response Management for Smart Grids With Wind Power," IEEE Transactions on Sustainable Energy, vol. 6, no. 2, pp. 625-634, 2015.

[12] M. Wei and J. Zhong, "Scenario-Based Real-Time Demand Response Considering Wind Power and Price Uncertainty," in 12th International Conference on the European Energy Market (EEM), Lisbon, 2015, pp. 1-5. [Online]. Available: http://ieeexplore.iee.. rg/stamp/stamp.jsp?tp=\&arnumber $=7216740$

[13] J. Dobschinski, "How good is my forecast ? Comparability of wind power forecast erros," in 13th International Workshop on Large Scale Integration of Wind Power into Power Systems as well as on Transmission Networks for Offshore Wind Farms, no. November, Berlin, 2014.

[14] M. H. Albadi and E. F. El-Saadany, "A summary of demand response in electricity markets," Electric Power Systems Research, vol. 78, no. 11, pp. 1989-1996, 2008.

[15] U.S. Department of Energy, "Benefits of Demand Response in Electricity Markets and Recommendations for Achieving Them," Department of Energy (DOE), Tech. Rep. February, 2006.

[16] M. G. Lijesen, "The real-time price elasticity of electricity," Energy Economics, vol. 29, no. 2, pp. 249-258, 2007. [Online]. Available: http://linkinghub.elsevier.com/retrieve/pii/S0140988306001010 